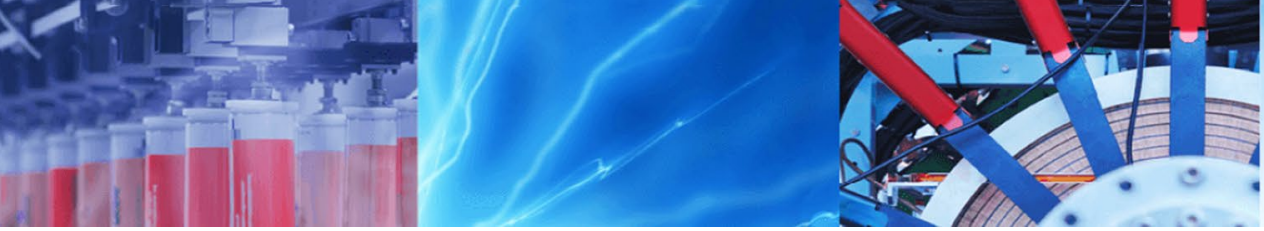

Research Article

\title{
Distributed finite-time active power sharing control with generation costs considered
}

\author{
Wenyu $\mathrm{He}^{1} \cdot \mathrm{Mei} \mathrm{Yu}^{1}$ (1) Junyan $\mathrm{Yu}^{2}$
}

Received: 26 June 2019 / Accepted: 21 November 2019 / Published online: 27 November 2019

(c) Springer Nature Switzerland AG 2019

\begin{abstract}
With the generation costs considered, a modified distributed active power sharing control scheme for the inverter-based microgrid is proposed in this paper. Inverters are regarded as agents that can exchange information with adjacent inverters over a communication network. Since the distributed generations in the microgrid are usually of different types, the total generation cost of the microgrid must be considered. Thus, this controller is more practical than traditional power sharing control scheme. Furthermore, a distributed finite-time active power sharing control strategy is proposed to share active power in a shorter period of time. Some sufficient conditions are derived to achieve active power sharing. Finally, simulations are presented to illustrate the validity of the theoretical results.
\end{abstract}

Keywords Power sharing · Generation costs · Distributed control · Finite-time consensus

\section{List of symbols}

$C_{i} \quad$ Generation cost of DG $i$

$P_{i, \max }$ Maximum value of DG i's active power

$C_{i}^{\prime \prime} \quad$ Modified generation cost of DG $i$

$C_{i, \text { max }}^{\prime \prime}$ Maximum value of $C_{i}^{\prime \prime}$

$r_{i}$ The inverse of $P_{i, \text { max }}$ 's reciprocal

$R \quad$ Matrix composed of $r_{i}$

\section{Introduction}

To achieve flexible, reliable and economic power supply to local areas, the concept of microgrid is proposed, which is defined as various types of distributed generations (DGs), energy storage systems and a cluster of loads operated in coordination [1]. These different devices generate or storage variable frequency AC/DC power supplies and are connected to the synchronous $A C$ grid via a power electronics $D C / A C$ inverter [2]. The rapid growth of energy demand attracts the wide application of DGs in the power system.
These DG units play an essential role in mitigating pollution emission, reducing power transmission losses and improving local power utilization. Furthermore, the integrated microgrid is better in terms of supply quality, stability and reliability since it combines the complementary advantages of different types of DGs [3]. Nevertheless, the massive use of DG units also brings several challenges to the power system.

Besides the problem of voltage/frequency regulation and restoration [4], economic dispatch [5], and stateof-charge balancing of distributed energy storage system [6], the power sharing problem is a common issue in the operation of microgrids [7]. Power sharing can be described as the ability of local control of individual DG unit to achieve a desired distribution of power outputs of all power sources while meeting the load requirements in the grid. The practical significance of this control target is that it allows the utilization of the DGs in operation to be pre-specified [8].

$\triangle$ Mei Yu, meiyu@ncepu.edu.cn; Wenyu He, 1172227155@ncepu.edu.cn; Junyan Yu, jyyu09@gmail.com | 'School of Control and Computer Engineering, North China Electric Power University, Beijing 102206, People's Republic of China. ${ }^{2}$ School of Mathematical Sciences, University of Electronic Science and Technology of China, Chengdu, People's Republic of China. 
The traditional power sharing control of grid is based on centralized control scheme, and all the generations are controlled by a central controller which brings a complex network. In addition, a single-point failure may lead to the fault of the whole network, and this reduces the system reliability. Thus, to avoid this problem, decentralized control scheme was adopted in power sharing control [9]. However, for the large-scale microgrid, it is difficult to control the entire system if all the DGs operate independently. Then, to improve stability, scalability, and security of the microgrid, the power sharing scheme based on distributed control was presented, which used low-bandwidth communication to transmit information of variables [10].

In recent years, the research of power sharing control based on multi-agent consensus method has received more and more attention [11]. In these researches, inverters were regarded as agents that could exchange information with adjacent inverters over a communication network [12]. In [8], a consensus-based distributed voltage control was proposed, which solved the open problem of reactive power sharing in autonomous meshed inverter-based microgrids with inductive power lines. In [13], a distributed cooperative control scheme for largescale microgrids with switch communication topology and time-varying delays was presented. In the analysis of power sharing problem based on multi-agent consensus approach, convergence rate is also an important performance criteria that worth studying. Literature [14] proposed a finite-time controller to share the active power among DGs based on their active power ratings. However, they did not study the influence of generation costs. To reduce the total generation cost of the microgrid, power sharing control should be considered. In fact, power sharing in microgrid not only depends on rating of DGs, but also relates to generation costs [15].

In this paper, a modified distributed cooperative control scheme is designed to share active power, in which the consistency variable is redefined. The proposed protocol saves the total power generation cost of microgrids while achieving active power sharing. The objective of reducing total generation cost is realized by making generators with high generation costs produce less active power. In order to share power in a shorter period of time, a finitetime active power sharing control scheme is proposed in this paper. The stability of the system with the proposed controller is proved based on Lyapunov stability theory. Moreover, simulation results verify the effectiveness of the controller.

The rest of this paper is organized as follows. Section 2 presents the graph theory and generation cost function. In Sect. 3, the distributed control scheme based on finitetime theory is proposed. Simulation examples are given in
Sect. 4 to illustrate the effectiveness of the result. Conclusions are presented in Sect. 5.

Notations: Throughout this paper, $\|\cdot\|$ denotes the Euclidean norm for vectors and the induced 2-norm for matrices, respectively. $I_{n}$ denotes the $n$-dimensional identity matrix. $\mathbb{R}^{n}$ denotes the $n$-dimensional Euclidean space. $\mathbb{R}^{m \times n}$ denotes the set of all the $m \times n$ real matrices. For a given matrix or vector $A, A^{T}$ is its transpose. $\mathbf{1}_{n}=[1, \ldots, 1]^{T} \in \mathbb{R}^{n}$. diag $\{\cdot\}$ denotes a block diagonal matrix formed by its input. $\operatorname{sig}(\cdot)^{\alpha}=\operatorname{sign}(\cdot)|\cdot|^{\alpha}$ and $\operatorname{sign}()$ is the sign function.

\section{Problem formulation}

\subsection{Graph theory}

In this paper, the communication connections in the microgrid can be captured by an undirected communication graph $\mathcal{G}(\mathcal{A})=\{\mathcal{V}, \mathcal{E}, \mathcal{A}\}$, where $\mathcal{V}=\{1,2, \ldots, N\}$ is the set of nodes. The neighbor set of node $i$ is denoted by $\mathcal{N}_{i}=\{j \in \mathcal{V} \mid(i, j) \in \mathcal{E}\}$, where $\mathcal{E} \subset \mathcal{V} \times \mathcal{V}$ is the set of all edges between connected agents, and $\mathcal{A}=\left[a_{i j}\right] \in \mathbb{R}^{N \times N}$ is the weighted adjacency matrix with elements $a_{i j}=a_{j i}$, if $j \in \mathcal{N}_{i}, a_{i j}=1$; otherwise, $a_{i j}=0$. The Laplacian matrix of graph $\mathcal{G}(\mathcal{A})$ is defined as $L=\mathcal{D}-\mathcal{A}$, where $\mathcal{D}=\operatorname{diag}\left\{d_{1}, d_{2}, \cdots, d_{N}\right\}$ is called the degree matrix with $d_{i}=\sum_{j \in \mathcal{N}_{i}} a_{i j}$.

\subsection{Generation cost function}

In microgrid, there are several different types of DGs, like fuel generators, microturbine generators and solar generators. Despite of the various differences, all the distributed generators have the same cost function, which can be denoted by following expression:

$C_{i}=b_{i}+C_{m, i}+C_{l, i}+C_{f, i}+C_{\xi, i}$

where $b_{i}$ is the levelized capital cost which is a constant term, and $C_{m, i}, C_{l, i}, C_{f, i}$ and $C_{\xi, i}$ represent the maintenance cost, distribution cost related to feeder power losses, fuel cost, and greenhouse emission penalty/incentive [15], which depend on the active power of DG i. In [15], to compare per-unit generation costs of different DGs based on the DG maximum powers, the generation costs curves are normalized to:

$C_{i}^{\prime}=\frac{C_{i}}{P_{i, \max }}$,

where $P_{i, \max }$ is the maximum value of $P_{i}(t)$, and $P_{i}(t)$ is the active power of DG $i$. Let 
$C_{i, 0}^{\prime}=\left.C_{i}^{\prime}\right|_{P_{i}^{\prime}=0}, P_{i}^{\prime}=\frac{P_{i}(t)}{P_{i, \max }} ;$

$C_{i}^{\prime \prime}=C_{i}^{\prime}-C_{i, 0}^{\prime}$

Then the constant term in $C_{i}^{\prime}$ is removed since it is independent of power generation. To illustrate the difference of the generation cost curves (2) and (4), three different DGs are randomly selected in this paper. A microgrid consisting of these three DGs is shown in Fig. 1a, where DG 1, DG 2 and DG 3 are assumed to be fuel generator, microturbine generator and solar generator with there own inverters. Furthermore, the normalized cost curves of the three types of DGs are shown in Fig. 1b, and the modified cost curves are shown in Fig. 1c.

\section{Active power sharing control scheme}

In this paper, it is assumed that $P_{i}(t)$ can be measured by sensor and the active power signal is transmitted to all its communication neighbors. Inverters are regarded as agents that can exchange information with adjacent inverters over a communication network. In general, the active power control scheme is designed as [13]:

$\dot{P}_{i}(t)=u_{i}(t)$

where $u_{i}(t)$ is the consensus controller designed as:

$u_{i}(t)=\sum_{j \in \mathcal{N}_{i}} a_{i j}\left(r_{i} P_{i}(t)-r_{j} P_{j}(t)\right)$,

$r_{i}=-\frac{1}{P_{i, \max }}, i=1,2, \ldots, N$.

To reduce the total generation cost $m=\sum_{i=1}^{N} C_{i}$ of the microgrid, the control of power sharing should be related to the costs of each generator [15]. In this paper, a new controller for active power sharing is proposed:

$\dot{P}_{i}(t)=u_{i}(t)$,

$u_{i}(t)=\sum_{j \in \mathcal{N}_{i}} a_{i j}\left(r_{i} P_{i}(t)-r_{j} P_{j}(t)+\Delta_{i}-\Delta_{j}\right)$,

$\Delta_{i}=\Delta * C_{i, \max }^{\prime \prime}, i=1,2, \ldots, N$,

where $C_{i, \max }^{\prime \prime}=\left.C^{\prime \prime}\left(P_{i}\right)\right|_{P_{i}=P_{i, \max }}$, and $\Delta$ is a negative gain coefficient.

\subsection{Stability analysis}

In this section, the Lyapunov stability theory is used to analyse the stability of the active power sharing control scheme (9).

Lemma 1 [16] For a connected undirected graph $\mathcal{G}(\mathcal{A})$, the Laplacian matrix $L$ of $\mathcal{G}(\mathcal{A})$ has the following properties:

(1) $x^{T} L x=\frac{1}{2} \sum_{i, j=1}^{N} a_{i j}\left(x_{i}-x_{j}\right)^{2}$;

(2) Eigenvalue 0 of the graph Laplacian $L$ is algebraically simple and all the other eigenvalues are positive;

(3) The eigenvalues of $L$ can be denoted by $0=\lambda_{1}<\lambda_{2} \leq \cdots \leq \lambda_{N}$. In addition, $x^{T} L x \geq \lambda_{2} x^{\top} x$, where $x \neq 0, \mathbf{1}_{N}^{T} x=0$.

Denote

$x_{i}(t)=r_{i} P_{i}(t)+\Delta_{i}$,

where $r_{i}$ is defined in Eq. (7), hence the system (8) can be described as:

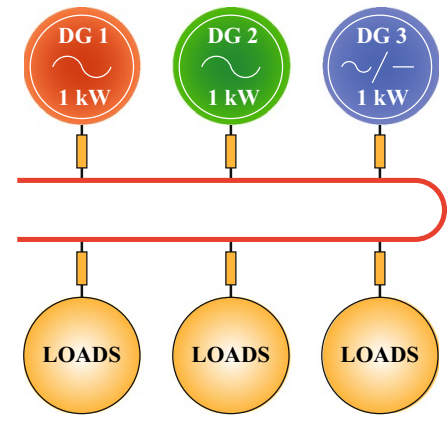

(a) Microgrid with three types of DGs

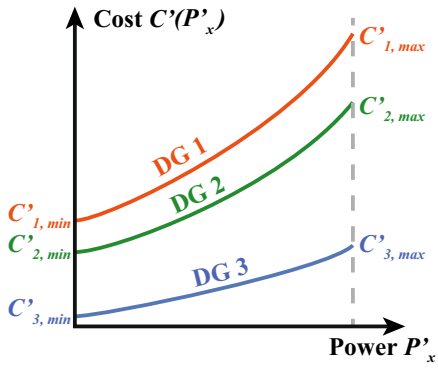

(b) Normalized generation cost curves

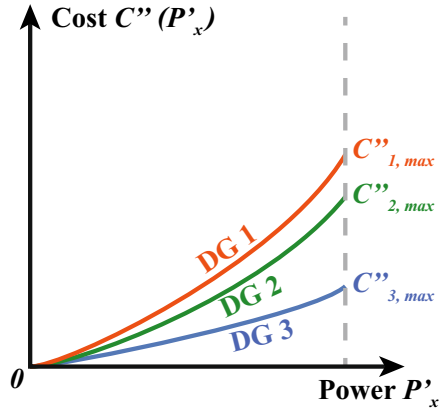

(c) Modified generation cost curves

Fig. 1 Illustration of microgrid with three DGs and their generation cost curves 
$\dot{x}_{i}(t)=r_{i} \sum_{j \in \mathcal{N}_{i}} a_{i j}\left(x_{i}(t)-x_{j}(t)\right)$.

Note that the consistent value of the above control strategy is different with that of the traditional control strategy. The extra item $\Delta_{i}$ is a negative coefficient which is related to generation costs. That is to say, generators with high generation costs produce less active power and vice versa. Therefore, the total generation cost is reduced for the whole microgrid.

Define $\quad x(t)=\left[x_{1}(t), x_{2}(t), \ldots, x_{N}(t)\right]^{T}, \quad$ and $x(0)=\left[x_{1}(0), \ldots, x_{N}(0)\right]^{T}$, then

$\dot{x}(t)=R L x(t)$,

where $R=\operatorname{diag}\left\{r_{1}, r_{2}, \ldots, r_{N}\right\}$, and $L$ is the Laplacian matrix of the communication graph $\mathcal{G}(\mathcal{A})$ defined in the previous section.

Lemma 2 Suppose $L$ is the Laplacian of an undirected, connected graph $\mathcal{G}$. There exist a non-negative left eigenvector associated with eigenvalue 0 of $R L$.

Proof Denotes $q=\left[q_{1}, \ldots, q_{N}\right]^{T}$ as a left eigenvector associated with eigenvalue 0 of $R L$. Then, one has $q^{T} R L=0$. Note that $\mathbf{1}_{N}^{T} L=0$, it can be concluded that $q^{T} R=\mathbf{1}_{N^{\prime}}^{T}$ which means $q^{T}=\mathbf{1}_{N}^{T} R^{-1}$. Since $r_{i}<0, q^{T}$ is negative. Without loss of generality, $q^{T}$ can be chosen as

$q^{T}=\frac{1}{\sum_{i=1}^{N} \frac{1}{r_{i}}} \mathbf{1}_{N}^{T} R^{-1}$.

It follows that $q_{i}>0$ and $\sum_{i=1}^{N} q_{i}=1$.

Due to $q^{T} R L=0$ and Eq. (13), one has $q^{T} \dot{x}(t)=0$. Hence, $q^{T} x(t)$ is an invariant quantity, which means that $b \mathbf{1}_{N}$ is the equilibrium point of system (13), where $b=\sum_{i=1}^{N} q_{i} x_{i}(0)$. Thus, $x(t)$ can be decomposed as:

$x(t)=b \mathbf{1}_{N}+\delta(t)$

where $\delta(t)=\left[\delta_{1}(t), \delta_{2}(t), \ldots, \delta_{N}(t)\right]^{T}$ is the group disagreement vector which satisfies $q^{T} \delta(t)=0[16]$. Since $L \mathbf{1}_{N}=0$, combining Eqs. (13) and (15), the system dynamics can also be described as:

$\dot{\delta}(t)=R L \delta(t)$.

Lemma 3 [17] For a connected undirected graph $\mathcal{G}(\mathcal{A})$ with Laplacian matrix L, it follows that

$\lambda_{2}=\min _{x^{T} q=0, x \neq 0} \frac{x^{T} L x}{x^{T} B x}$

where $q$ is defined in Lemma 2 , and $B=\operatorname{diag}\left\{q_{1}, \ldots, q_{N}\right\}$.
From the above Lemmas, the active power sharing can be realized by the proposed controller (9) based on the multiagent consensus approach.

Theorem 1 Considering a active power sharing system (8) with graph $\mathcal{G}(\mathcal{A})$ being connected and undirected, the $D G$ s with the designed controller (9) can share active power with generation costs considered.

Proof Since $r_{i}<0$, the matrix $R$ is negative definite, which means $-R$ is positive definite matrix. Hence, the Lyapunov functional candidate can be chosen as:

$V(t)=\frac{1}{2} \delta^{T}(t)\left(-R^{-1}\right) \delta(t)$,

where $R^{-1}$ is the inverse matrix of $R$. Calculating the time derivative of the Lyapunov function $V$, one has

$$
\begin{aligned}
\dot{V}(t) & =\delta^{T}(t)\left(-R^{-1}\right) \dot{\delta}(t) \\
& =\delta^{T}(t)\left(-R^{-1}\right) R L \delta(t) \\
& =-\delta^{T}(t) L \delta(t) .
\end{aligned}
$$

From Lemmas 2 and 3, it can be got that:

$$
\begin{aligned}
\dot{V}(t) & \leq-\lambda_{2} \delta^{T}(t) B \delta(t) \\
& =\lambda_{2} \frac{1}{\sum_{i=1}^{N} \frac{1}{r_{i}}} \delta^{T}(t)\left(-R^{-1}\right) \delta(t) \\
& =-2 s \lambda_{2} V<0,
\end{aligned}
$$

where $s=-1 /\left(\sum_{i=1}^{N} 1 / r_{i}\right)>0$.

This shows that $\delta(t)$ will converge to 0 as $t \rightarrow \infty$, ie,

$\lim _{t \rightarrow \infty}\left(\left(r_{i} P_{i}(t)+\Delta_{i}\right)-\left(r_{j} P_{j}(t)+\Delta_{j}\right)\right)=0$

for any $i$ and $j$, which means the DGs with the designed controller (9) can share active power with generation costs considered.

This completes the proof.

Under the distributed power sharing control strategy, the voltage value and output power information of each DGs are exchanged between the inverters which act as agents. Through the coordination and cooperation between the agents, the DGs can share active power with generation costs considered, ie, $\lim _{t \rightarrow \infty}\left(\left(r_{i} P_{i}(t)+\Delta_{i}\right)-\left(r_{j} P_{j}(t)+\Delta_{j}\right)\right)=0$. From the definition $\stackrel{t \rightarrow \infty}{\text { of } \Delta_{i / j} \text {, it can be obtained that DGs with high generation }}$ costs produce less active power and vice versa. Thus, the total generation cost can be reduced, which meet the demand of microgrid. 


\subsection{Finite-time consensus}

In this section, to share active power in a shorter period of time, the finite-time control protocol for active power sharing is proposed. Here, several important lemmas are listed as follows.

Lemma 4 [18] Suppose function $\phi: \mathbb{R}^{2} \rightarrow \mathbb{R}$ satisfies $\phi\left(x_{i}, x_{j}\right)=-\phi\left(x_{j}, x_{i}\right), i, j \in N, i \neq j$. Then for any undirected graph $\mathcal{G}$ and a set of numbers $y_{1}, y_{2}, \ldots, y_{N}$ :

$\sum_{i=1}^{N} \sum_{j \in \mathcal{N}_{i}} a_{i j} y_{i} \phi\left(x_{i}, x_{j}\right)=\frac{1}{2} \sum_{(i, j) \in \mathcal{E}} a_{i j}\left(y_{i}-y_{j}\right) \phi\left(x_{i}, x_{j}\right)$

Lemma 5 [19] For $x_{i} \in \mathbb{R}, i=1, \ldots, n, 0<p \leq 1$, then

$\left(\sum_{i=1}^{n}\left|x_{i}\right|\right)^{p} \leq \sum_{i=1}^{n}\left|x_{i}\right|^{p}$

Lemma 6 [20] Suppose that function $V(t):[0, \infty) \rightarrow[0, \infty)$ is differentiable (the derivative of $V(t)$ at 0 is in fact its right derivative) and

$\frac{\mathrm{d} V(t)}{\mathrm{d} t} \leq-K V(t)^{\mu}$

where $K>0$ and $0<\mu<1$. Then $V(t)$ will reach zero in finite time $t^{*} \leq V(0)^{1-\mu} /(K(1-\mu))$ and $V(t)=0$ for all $t \geq t^{*}$.

Then from all the Lemmas presented in this paper, the conclusion of active sharing in finite time is given as following.

Theorem 2 Considering the active power sharing system (8) with graph $\mathcal{G}(\mathcal{A})$ being connected and undirected, the DGs can share active power with generation costs considered in finite time with the designed controller (25).

$u_{i}(t)=\sum_{j \in \mathcal{N}_{i}} a_{i j} \operatorname{sig}\left(r_{i} P_{i}(t)-r_{j} P_{j}(t)+\Delta_{i}-\Delta_{j}\right)^{\alpha}$,

where $\alpha$ is a constant which satisfies $0<(\alpha+1) / 2<1$.

Proof It can be obtained from Eq. (11) that

$\dot{x}_{i}(t)=r_{i} \sum_{j \in \mathcal{N}_{i}} a_{i j} \operatorname{sig}\left(x_{i}(t)-x_{j}(t)\right)^{\alpha}$.

Furthermore, since $L \mathbf{1}_{N}=0$, from Eq. (15), Eq. (26) can be described as:

$\dot{\delta}_{i}(t)=r_{i} \sum_{j \in \mathcal{N}_{c}} a_{i j} \operatorname{sig}\left(\delta_{i}(t)-\delta_{j}(t)\right)^{\alpha}$.
Because $r_{i}<0$, one can choose the following Lyapunov functional candidate:

$V(t)=\frac{1}{2} \sum_{i=1}^{N}\left(-\frac{1}{r_{i}}\right) \delta_{i}^{2}(t)$

Calculating the time derivative of the Lyapunov function $V(t)$, one has

$$
\begin{aligned}
\dot{V}(t) & =\sum_{i=1}^{N} \delta_{i}(t)\left(-\frac{1}{r_{i}}\right) \dot{\delta}_{i}(t) \\
& =-\sum_{i=1}^{N} \delta_{i}(t) \sum_{j \in \mathcal{N}_{c}} a_{i j} \operatorname{sig}\left(\delta_{i}(t)-\delta_{j}(t)\right)^{\alpha},
\end{aligned}
$$

by Lemma 4,

$$
\begin{aligned}
\dot{V}(t) & =-\sum_{i=1}^{N} \delta_{i}(t) \sum_{j \in \mathcal{N}_{c}} a_{i j} \operatorname{sig}\left(\delta_{i}(t)-\delta_{j}(t)\right)^{\alpha} \\
& =-\frac{1}{2} \sum_{(i, j) \in \mathcal{E}} a_{i j}\left(\delta_{i}(t)-\delta_{j}(t)\right) \operatorname{sig}\left(\delta_{i}(t)-\delta_{j}(t)\right)^{\alpha} \\
& =-\frac{1}{2} \sum_{(i, j) \in \mathcal{E}}\left(a_{i j}\right)\left|\delta_{i}(t)-\delta_{j}(t)\right|^{\alpha+1} \\
& =-\frac{1}{2} \sum_{(i, j) \in \mathcal{E}}\left(\left(a_{i j}\right)^{\frac{1}{\alpha+1}}\left|\delta_{i}(t)-\delta_{j}(t)\right|\right)^{\alpha+1} \\
& =-\frac{1}{2} \sum_{(i, j) \in \mathcal{E}}\left(\left(a_{i j}\right)^{\frac{2}{\alpha+1}}\left|\delta_{i}(t)-\delta_{j}(t)\right|^{2}\right)^{\frac{\alpha+1}{2}}
\end{aligned}
$$

then by Lemma 5,

$$
\begin{aligned}
\dot{V}(t) & =-\frac{1}{2} \sum_{(i, j) \in \mathcal{E}}\left(\left(a_{i j}\right)^{\frac{2}{\alpha+1}}\left|\delta_{i}(t)-\delta_{j}(t)\right|^{2}\right)^{\frac{\alpha+1}{2}} \\
& \leq-\frac{1}{2}\left(\sum_{(i, j) \in \mathcal{E}}\left(a_{i j}\right)^{\frac{2}{\alpha+1}}\left|\delta_{i}(t)-\delta_{j}(t)\right|^{2}\right)^{\frac{\alpha+1}{2}} .
\end{aligned}
$$

Denote $H=\left[a_{i j}^{\frac{2}{\alpha+1}}\right] \in \mathbb{R}^{N \times N}$, it can be concluded that $\mathcal{G}(H)$ is also connected and undirected. From Lemma 3, one has

$$
\begin{aligned}
\dot{V}(t) & \leq-\frac{1}{2}\left(2 \delta(t)^{T} \tilde{L} \delta(t)\right)^{\frac{\alpha+1}{2}} \\
& \leq-\frac{1}{2}\left(2 \tilde{\lambda}_{2} \delta(t)^{T} B \delta(t)\right)^{\frac{\alpha+1}{2}} \\
& =-\frac{1}{2}\left(2 s \tilde{\lambda}_{2} \delta(t)^{T}\left(-R^{-1}\right) \delta(t)\right)^{\frac{\alpha+1}{2}},
\end{aligned}
$$

where $\tilde{L}$ is the graph Laplacian of $\mathcal{G}(H)$ and $\tilde{\lambda}_{2}$ is the second smallest eigenvalue of $\mathcal{G}(H)$. It follows that

$$
\begin{aligned}
\dot{V}(t) & \leq-\frac{1}{2}\left(2 s \tilde{\lambda}_{2} V(t)\right)^{\frac{\alpha+1}{2}} \\
& =-2^{\frac{\alpha-1}{2}}\left(s \tilde{\lambda}_{2}\right)^{\frac{\alpha+1}{2}} V(t)^{\frac{\alpha+1}{2}} .
\end{aligned}
$$


Then, it can be got that $\dot{V}(t)+K V(t)^{\frac{\alpha+1}{2}} \leq 0$, where $K=2^{\frac{\alpha-1}{2}}\left(s \tilde{\lambda}_{2}\right)^{\frac{\alpha+1}{2}}$, and $0<(\alpha+1) / 2<1$. Thus, from Lemma 6 , it can be concluded that the Lyapunov function $V(t)$ will reach zero in finite time:

$t_{0} \leq \frac{V(0)^{(1-\alpha) / 2}}{\left(2^{\frac{\alpha-3}{2}}\left(s \tilde{\lambda}_{2}\right)^{\frac{\alpha+1}{2}}\right)(1-\alpha)}$.

Furthermore, one has $x_{i}=x_{j}$ for any $i$ and $j$ while $V(t)$ reach zero, which means:

$r_{i} P_{i}(t)+\Delta_{i}=r_{j} P_{j}(t)+\Delta_{j}$

for any $i$ and $j$. That is to say the DGs with controller (25) can share active power with generation costs considered in finite time.

This completes the proof.

Under the finite-time control protocol (25), the voltage value and output power information of each DGs are exchanged between the inverters which act as agents. Through the coordination and cooperation between the agents, the active power sharing time of DGs can be guaranteed in finite time, and this meet the needs of microgrid.

Remark 1 Denote $\Omega=1 /\left(4 s \tilde{\lambda}_{2}\right)$. If $0<V(0) \leq \Omega$, calculating the derivative of time $t_{0}$ with respect to $\alpha$, one has $\dot{t}_{0}>0$ for any $0<\alpha<1$. Thus, if $0<V(0) \leq \Omega$ and $\alpha$ increases in $(0,1), t_{0}$ also increases. Above arguments show that smaller $\alpha$ lead to a higher convergence rate when the agents' states satisfy $0<V(0) \leq \Omega$.

\section{Simulation and analysis}

In this section, numerical examples will be given to demonstrate the effectiveness of our main results. The networked system consists of 5 DGs operating in parallel and supplying a variable load, these DGs are connected to main grid via 5 inverters, respectively, which is shown in Fig. 2. The communication network of the inverter group is shown in Fig. 3. Its adjacency matrix $\mathcal{A}$ is given as

$\mathcal{A}=\left[\begin{array}{lllll}0 & 1 & 1 & 0 & 1 \\ 1 & 0 & 0 & 1 & 1 \\ 1 & 0 & 0 & 1 & 1 \\ 0 & 1 & 1 & 0 & 0 \\ 1 & 1 & 1 & 0 & 0\end{array}\right]$

The corresponding Laplacian matrix $L$ is given as

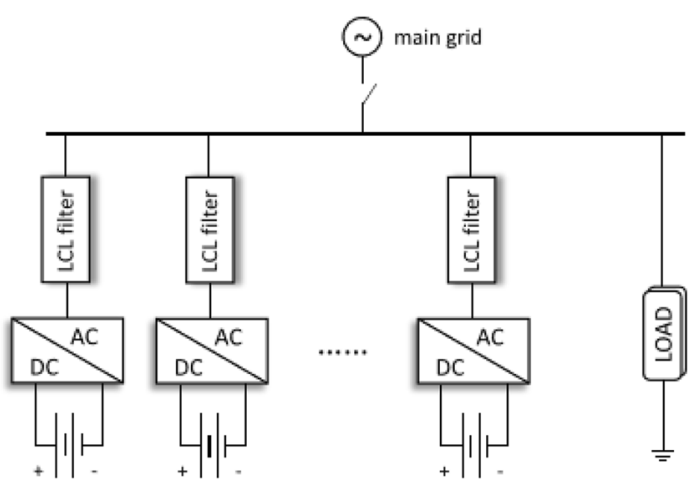

Fig. 2 Microgrid with parallel operating DGs interfaced by inverters

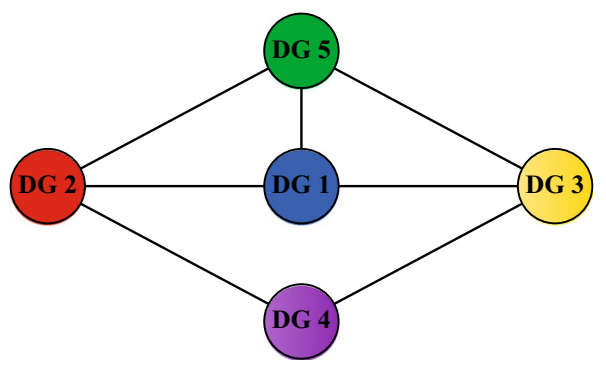

Fig. 3 Communication structure of the DGs

$L=\left[\begin{array}{ccccc}3 & -1 & -1 & 0 & -1 \\ -1 & 3 & 0 & -1 & -1 \\ -1 & 0 & 3 & -1 & -1 \\ 0 & -1 & -1 & 2 & 0 \\ -1 & -1 & -1 & 0 & 3\end{array}\right]$

In this microgrid, the nominal voltage amplitude and frequency of the microgrid are $200 \mathrm{~V}$ and $50 \mathrm{~Hz}$, respectively. Each inverter's maximum active power is assumed to be $P_{\text {max }}=(1,0.8,1,0.8,1) \mathrm{kW}$, and the initial active power is set as $P_{i}(0)=0.5 \mathrm{~kW}$. The $5 \mathrm{DGs}$ are assumed to be diesel generator, microturbine generator, microturbine generator, diesel generator and solar generator, respectively. The modified generation cost functions of the five DGs are given as

$$
\begin{aligned}
C^{\prime \prime}\left(P_{1}\right)= & 0.04 P_{1}+0.02\left(8 P_{1}+25 P_{1}^{2}\right)+0.1\left(-3.92 P_{1}+5.638 P_{1}^{2}\right) \\
& +10^{-4} e^{8.333 P_{1}}
\end{aligned}
$$

Table 1 Each DG's $P_{\max }$ and generation cost associated to $P_{\max }$

\begin{tabular}{llllll}
\hline DG & 1 & 2 & 3 & 4 & 5 \\
\hline$P_{\text {max }}(k W)$ & 1 & 0.8 & 1 & 0.8 & 1 \\
Cost & 0.91 & 0.48 & 0.68 & 0.67 & 0.19 \\
\hline
\end{tabular}




$$
\begin{aligned}
C^{\prime \prime}\left(P_{2}\right)= & \left(0.04 P_{2}+0.02\left(10 P_{2}+16 P_{2}^{2}\right)+0.1\left(-5.371 P_{2}+6.49 P_{2}^{2}\right)\right. \\
& \left.+4 \times 10^{-4} e^{2.857 P_{2}}\right) / 0.8
\end{aligned}
$$

$$
\begin{aligned}
C^{\prime \prime}\left(P_{3}\right)= & 0.04 P_{3}+0.02\left(10 P_{3}+16 P_{3}^{2}\right)+0.1\left(-5.371 P_{3}+6.49 P_{3}^{2}\right) \\
& +4 \times 10^{-4} e^{2.857 P_{3}}
\end{aligned}
$$

$$
C^{\prime \prime}\left(P_{4}\right)=\left(0.04 P_{4}+0.02\left(8 P_{4}+25 P_{4}^{2}\right)+0.1\left(-3.92 P_{4}+5.638 P_{4}^{2}\right)\right.
$$$$
\left.+10^{-4} e^{8.333 P_{4}}\right) / 0.8 \text {; }
$$

$C^{\prime \prime}\left(P_{5}\right)=0.2\left(P_{5}+0.1\left(-0.1 P_{5}+0.1 P_{5}^{2}\right)\right)$.

Then, by the above functions, each generation cost associated with its maximum active power is $C_{\max }^{\prime \prime}=(0.91,0.48,0.68,0.67,0.19)$ and is shown in Table 1.

Case study 1 : In this case, the active power sharing system is studied based on controller (6) and controller (9).

Figure 4 shows the trajectories of the active power of each DG under the controller without generation costs. From Fig. 4, it can be conclude that the proportional active power sharing can be achieved. Furthermore, each DG's active power in Fig. 4 is $P=(0.543,0.435,0.543,0.435,0.543) \mathrm{kW}$. Its corresponding generation cost is $C^{\prime \prime}=(0.210,0.069,0.126,0.148,0.108)$, and the total generation cost is 0.661 . Note that the curves of DG 1 and DG 5 are coincident. There are two reasons for this: 1. The maximum active powers of DG1 and DG5 are equal; 2 . From Fig. 3, it can be obtained that the positions of the two DGs are symmetrical, which means the information received from the other agents in the network is identical. Thus, control signals of the two agents are the same and this leads to the coincident curves.

For the DGs under the control of scheme (9), each example is divided into two cases: $1: \Delta=-0.1 ; 2$ : $\Delta=-0.25$. Figures 5 and 6 show the trajectories of the active power of each DG under the controller (9). One can notice that the two active power sharing with generation costs considered are achieved. These are consistent with Theorem 1. Moreover, it can be got that all the active powers have changed compared to those in Fig. 4. In addition, some of them become bigger and the other become smaller, which are related to their own generation costs. The magnitudes of the changes are directly related to the value of $\Delta$. The active powers in Figs. 5 and 6 are $P=(0.511,0.443,0.534,0.428,0.582) \mathrm{kW}$ and $P=(0.463,0.456,0.521,0.419,0.641) \mathrm{kW}$, respectively. These show that generators with high generation costs produce less active power and vice versa. Their corresponding generation costs are $C^{\prime \prime}=(0.180,0.075,0.120,0.141,0.116)$ and $C^{\prime \prime}=(0.140,0.084,0.110,0.133,0.128)$, respectively. The total generation costs are 0.632 and 0.595 , respectively. Comparing the above results, it can be calculated that the modified control scheme saves $4.39 \%$ total generation cost in the case of $\Delta=-0.1$, and it saves $9.98 \%$ total generation cost while $\Delta=-0.25$. It can be concluded that the lager the absolute value of $\Delta$, the greater
Fig. 4 The trajectories under the control scheme (6)

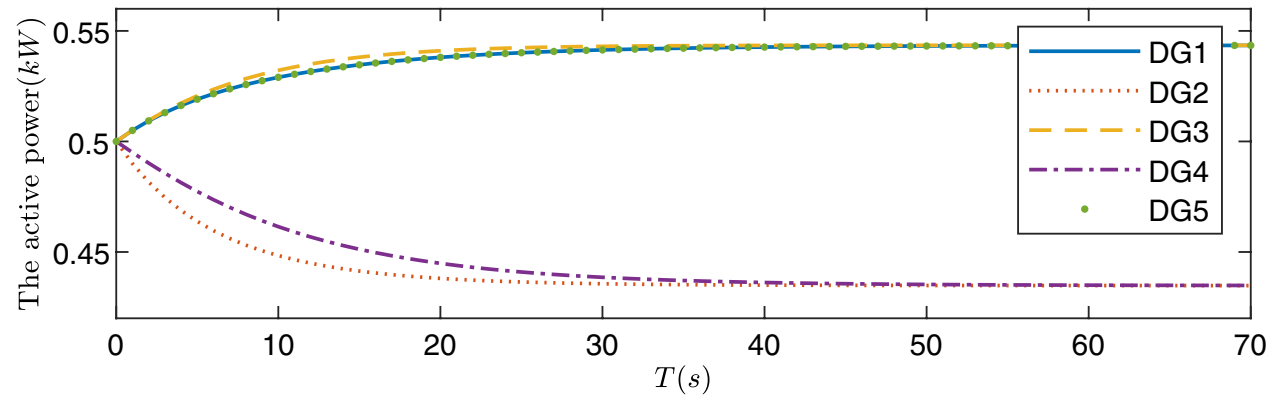

(a) The active powers

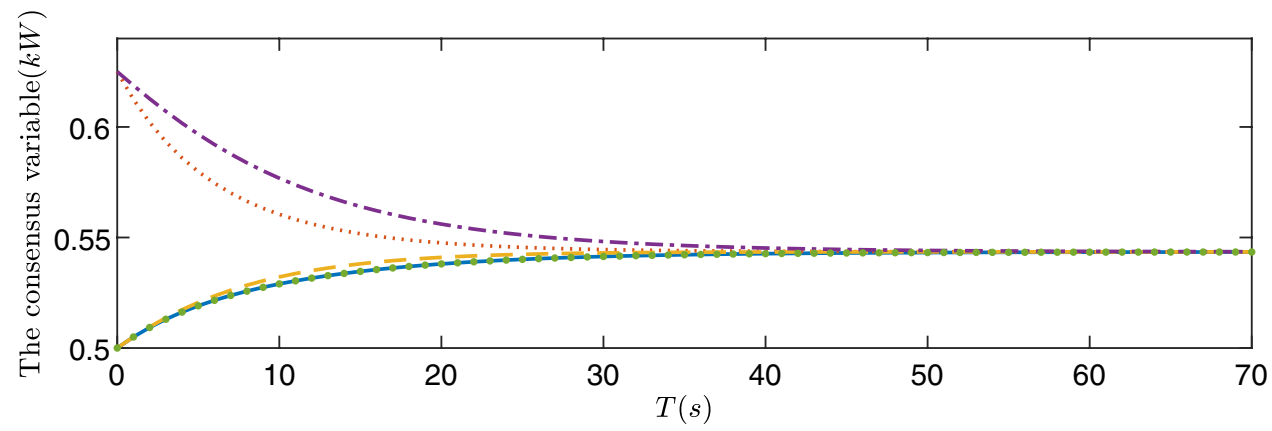

(b) The consensus variables 
Fig. 5 The trajectories under the control scheme (9) $(\Delta=-0.1)$
Fig. 6 The trajectories under the control scheme (9) $(\Delta=-0.25)$

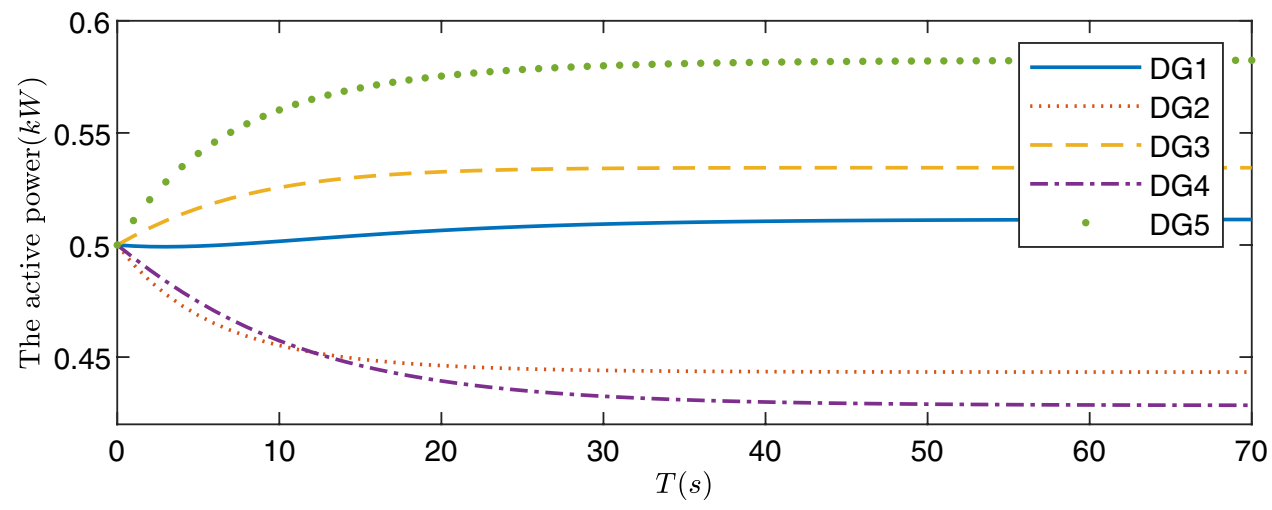

(a) The active powers

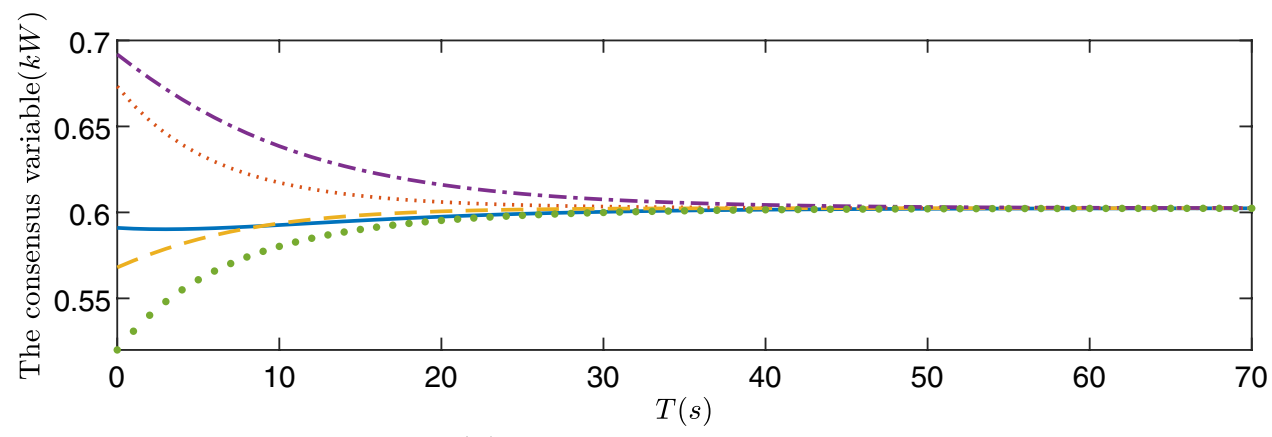

(b) The consensus variables

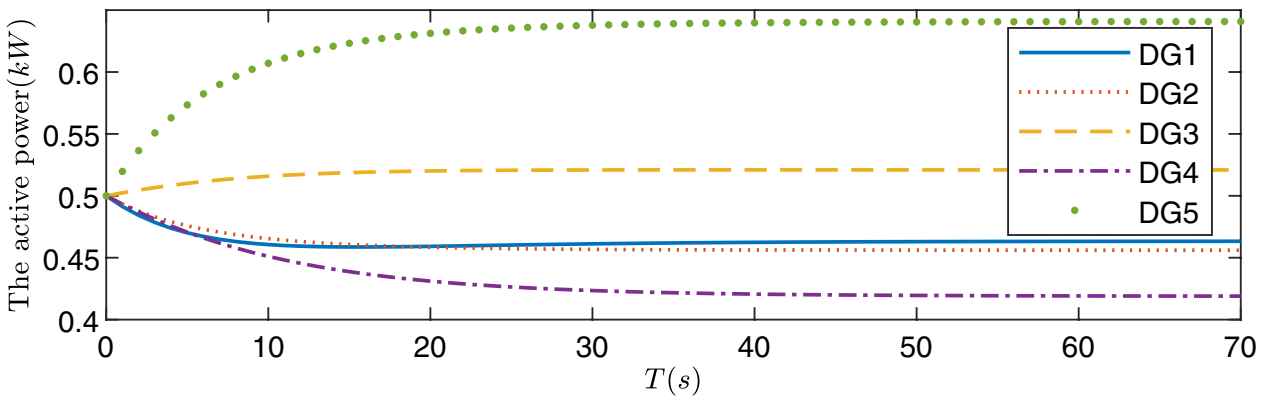

(a) The active powers

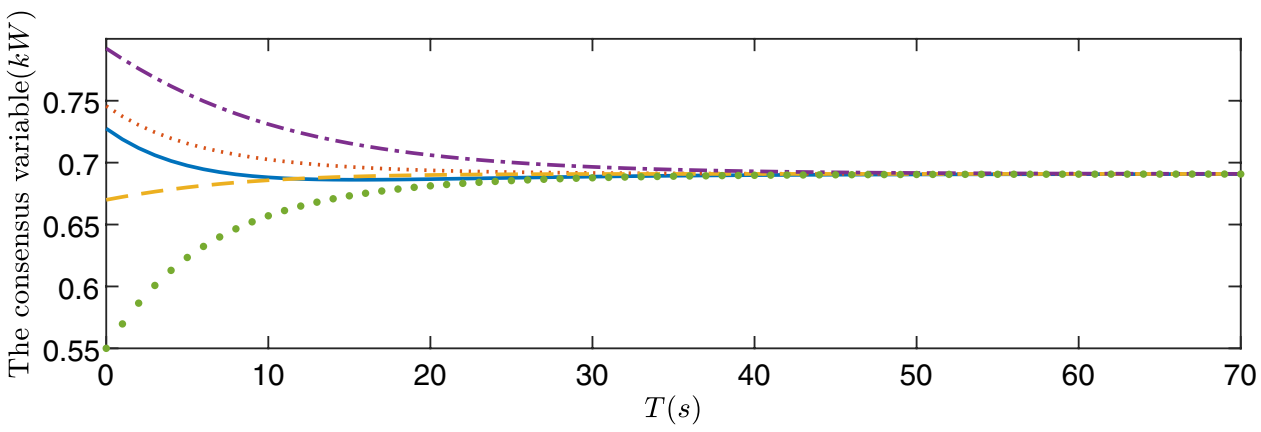

(b) The consensus variables

For the DGs under the control of scheme (25), two cases have been simulated in this paper: $1: \alpha=0.9 ; 2$ : $\alpha=0.8$. Figures 7 and 8 show the trajectories of the active power of each DG under the controller (25). It 
Fig. 7 The trajectories under the finite-time control scheme (25) $(\alpha=0.9)$
Fig. 8 The trajectories under the finite-time control scheme (25) $(\alpha=0.8)$

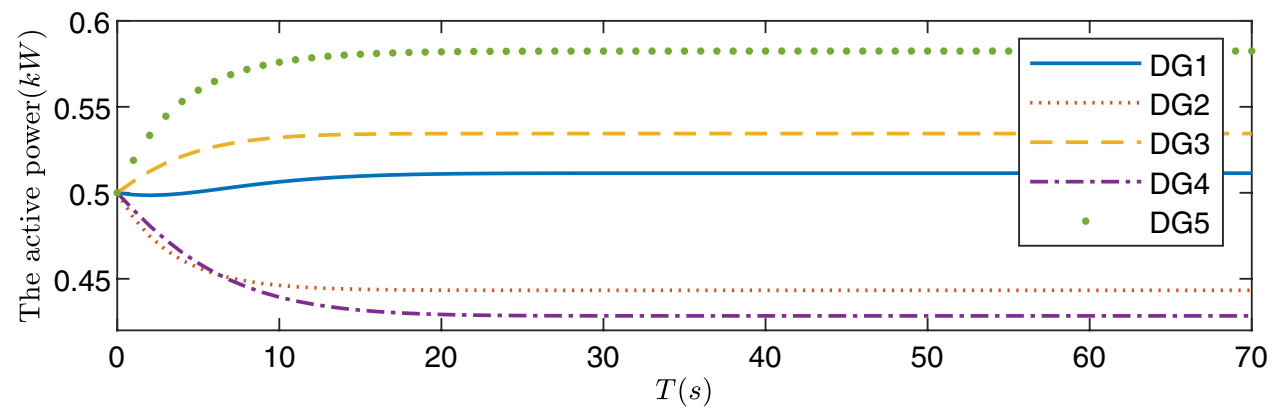

(a) The active powers

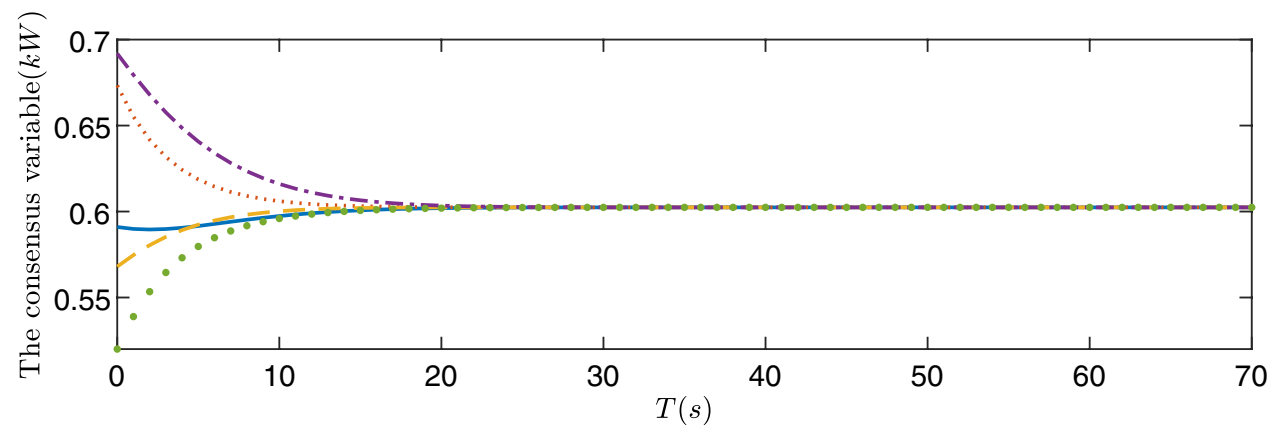

(b) The consensus variables

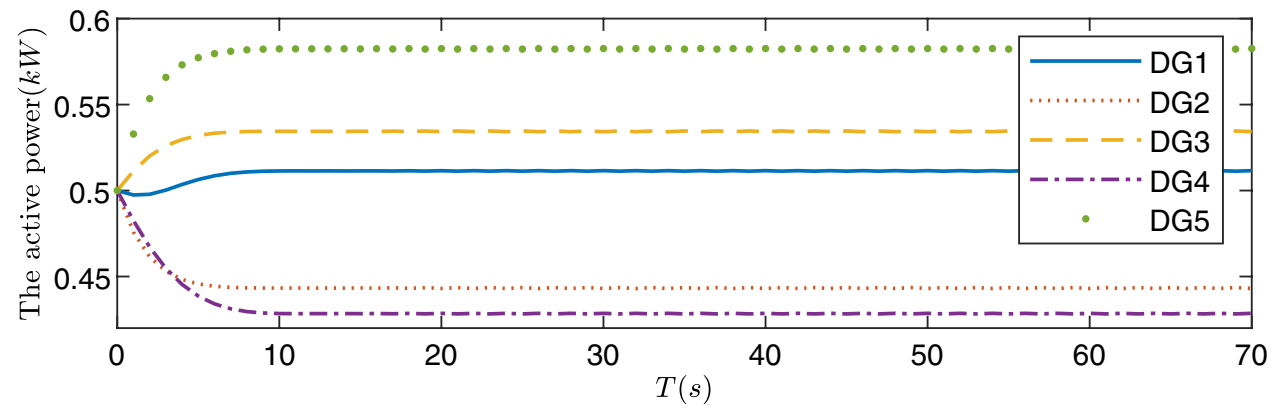

(a) The active powers

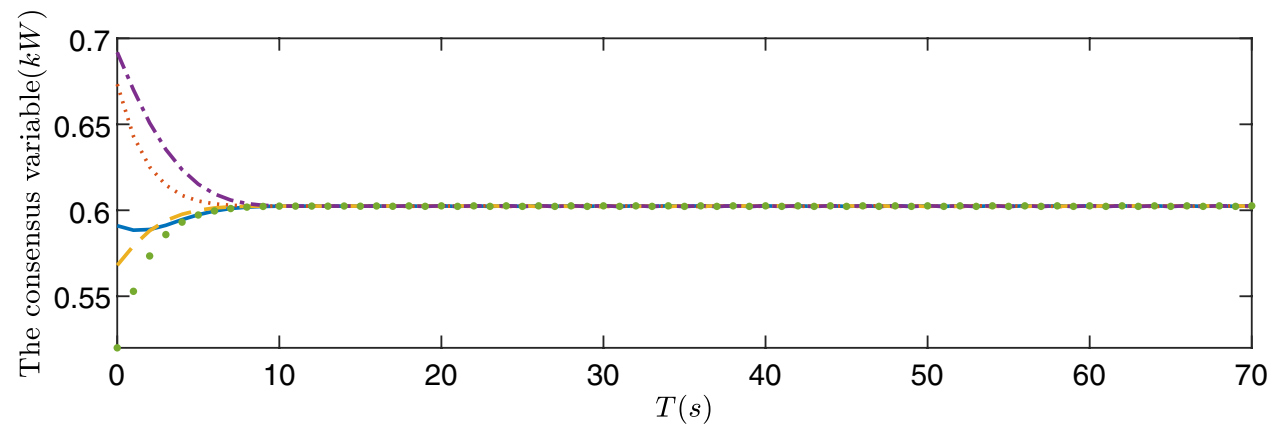

(b) The consensus variables

can be got that the two power sharings with generation costs considered are achieved. In addition, comparing Figures 5, 7 and 8, it is obvious to see that the control scheme significantly reduces the convergence time. The convergence time under finite-time control in Fig. $7(\alpha=0.9)$ is about half of that of Fig. 4 , and the convergence time with Fig. $8(\alpha=0.8)$ is about half of that of Fig. $7(\alpha=0.9)$, which is consistent with Theorem 2 . Accordingly, by appropriately selecting the value of $\alpha$, control scheme (25) can effectively reduce the 
convergence time on the basis of ensuring stable operation of microgrid active power sharing system.

\section{Conclusion}

A modified distributed active power sharing control scheme with generation costs of DGs considered has been presented in this paper. Inverters are regarded as agents that could exchange information with adjacent inverters over a communication network. Unlike conventional power sharing controller which is only based on power rating, the proposed controller in this paper is more practical and meaningful for the microgrid since it reduces the total generation cost. Moreover, a distributed finite-time active power sharing control scheme has been proposed. Thus, the active power sharing time could be guaranteed to be finite time by the algorithm. The effectiveness of the two control schemes has been verified by simulation.

\section{Compliance with ethical standards}

Conflict of interest The authors declare that they have no conflict of interest.

\section{References}

1. Olivareset $D$ et al (2014) Trends in microgrid control. IEEE Trans Smart Grid 5(4):1905-1919. https://doi.org/10.1109/ TSG.2013.2295514

2. Majumder R, Chaudhuri B, Ghosh A, Majumder R, Ledwich G, Zare $F$ (2010) Improvement of Stability and Load Sharing in an Autonomous Microgrid Using Supplementary Droop Control Loop. IEEE Trans Power Syst 25(2):796-808. https://doi. org/10.1109/tpwrs.2009.2032049

3. Farhangi $\mathrm{H}$ (2010) The path of the smart grid. IEEE Power Energy Mag 8(1):18-28. https://doi.org/10.1109/MPE.2009.934876

4. Schiffer J, Ortega R, Astolfi A, Raisch J, Sezi T (2014) Conditions for stability of droop-controlled inverter-based microgrids. Automatica 50(10):2457-2469. https://doi.org/10.1016/j.autom atica.2014.08.009

5. Zhang Z, Chow M (2012) Convergence analysis of the incremental cost consensus algorithm under different communication network topologies in a smart grid. IEEE Trans Power Syst 27(4):1761-1768. https://doi.org/10.1109/tpwrs.2012.2188912

6. Mokhtari G, Nourbakhsh G, Ghosh A (2013) Smart Coordination of Energy Storage Units (ESUs) for Voltage and Loading Management in Distribution Networks. IEEE Trans Power Syst 28(4):4812-4820. https://doi.org/10.1109/TPWRS.2013.2272092

7. Li Y, Kao CN (2009) An accurate power control strategy for powerelectronics-interfaced distributed generation units operating in a lowvoltage multibus microgrid. IEEE Trans Power Electron 24(12):2977-2988. https://doi.org/10.1109/tpel.2009.2022828

8. Schiffer J, Seel T, Raisch J, Sezi T (2016) Voltage stability and reactive power sharing in inverter-based microgrids with consensusbased distributed voltage control. IEEE Trans Control Syst Technol 24(1):96-109. https://doi.org/10.1109/TCST.2015.2420622

9. Mohamed Y, Saadany E (2008) Adaptive decentralized droop controller to preserve power sharing stability of paralleled inverters in distributed generation microgrids. IEEE Trans Power Electron 23(6):2806-2816. https://doi.org/10.1109/ tpel.2008.2005100

10. Ahn S, Park J, Chung I, Moon S, Kang S, Nam S (2010) Power-sharing method of multiple distributed generators considering control modes and configurations of a microgrid. IEEE Trans Power Del 25(3):2007-2016. https://doi.org/10.1109/tpwrd.2010.20477 36

11. Lu L, Chu C (2015) Consensus-based droop control synthesis for multiple DICs in isolated micro-grids. IEEE Trans Power Syst 30(5):2243-2256. https://doi.org/10.1109/TPWRS.2014.2368135

12. Fan Y, Hu G, Egerstedt M (2017) Distributed reactive power sharing control for microgrids with event-triggered communication. IEEE Trans Control Syst Technol 25(1):118-128. https:// doi.org/10.1109/TCST.2016.2552982

13. Jingang $L$, Hong $Z$, Wenshan $H$, Xiaoqing $L$ (2015) Distributed secondary control for reactive power sharing of inverter-based DGs in microgrids. In: 2015 34th Chinese control conference (CCC), Hangzhou, pp 8990-8995. https://doi.org/10.1109/ChiCC .2015.7261062

14. Bidram A, Davoudi A Lewis F (2014) Finite-time frequency synchronization in microgrids. In: 2014 IEEE energy conversion congress and exposition (ECCE), Pittsburgh, PA, pp 2648-2654. https://doi.org/10.1109/ECCE.2014.6953756

15. Nutkani I, Loh P, Wang P, Blaabjerg F (2014) Autonomous droop scheme with reduced generation cost. IEEE Trans Ind Electron 61(12):6803-6811. https://doi.org/10.1109/TIE.2014.2314056

16. Olfati-Saber R, Murray R (2004) Consensus problems in networks of agents with switching topology and time-delays. IEEE Trans Autom Control 49(9):1520-1533. https://doi.org/10.1109/ tac. 2004.834113

17. Yu W, Chen G, Cao M, Kurths J (2010) Second-order consensus for multiagent systems with directed topologies and nonlinear dynamics. IEEE Trans Syst Man Cybern Part B (Cybernetics) 40(3):881-891. https://doi.org/10.1109/TSMCB.2009.2031624

18. Jiang $F$, Wang $L$ (2009) Finite-time information consensus for multi-agent systems with fixed and switching topologies. Physica D 238(16):1550-1560. https://doi.org/10.1016/j.physd .2009.04.011

19. Li S, Du H, Lin X (2011) Finite-time consensus algorithm for multi-agent system with double-integrator dynamics. Automatica 47(8):1706-1712. https://doi.org/10.1016/j.automatica .2011 .02 .045

20. Wang L, Xiao F (2010) Finite-time consensus problems for networks of dynamic agents. IEEE Trans Autom Control 55(4):950955. https://doi.org/10.1109/TAC.2010.2041610

Publisher's Note Springer Nature remains neutral with regard to jurisdictional claims in published maps and institutional affiliations. 